\title{
Effect of Added Garcinia Fruit on Total Phenolic Compound Content, Antioxidant Properties and Quality Changes of the Southern Sour Curry Paste, Keang-hleung, during Storage
}

\author{
Preeyaporn Promjiam $^{1}$, Sunisa Siripongvutikorn ${ }^{1,2^{*}}$, Worapong Usawakesmanee ${ }^{2}$, \\ Santad Wichienchot ${ }^{1}$ \\ ${ }^{1}$ Nutraceutical and Functional Food Research Center, Faculty of Agro-Industry, Prince of Songkla University, Songkhla, Thailand; \\ ${ }^{2}$ Department of Food Technology, Faculty of Agro-Industry, Prince of Songkla University, Songkhla, Thailand. \\ Email: ${ }^{*}$ sunisa.s@psu.ac.th, ${ }^{*}$ sunisasiripongvutikorn@gmail.com
}

Received May $8^{\text {th }}, 2013$; revised June $5^{\text {th }}, 2013$; accepted June $14^{\text {th }}, 2013$

Copyright (C) 2013 Preeyaporn Promjiam et al. This is an open access article distributed under the Creative Commons Attribution License, which permits unrestricted use, distribution, and reproduction in any medium, provided the original work is properly cited.

\begin{abstract}
The favorite soup for Thai particularly in the southern part is Southern sour curry or Keang-hleung soup. The ingredients used in the paste are turmeric rhizome, garlic, shallot and chili. However, for making the sour soup, the souring agent such as lime juice, tamarind pod or garcinia, fruit is added. This study aimed to compare quality changes, total phenolic compound and antioxidant properties of the pastes as affected of added garicia fruit during storage. It was found that the total phenolic compound content of basic paste without the garcinia, garcinia Keang-hleung paste and garcinia Keang-hleung paste without salt decreased as increased storage time. Moreover, the DPPH (2,2-diphenyl1-picrylhydrazyl) radical scavenging activity and the ferric reducing power (FRAP) activity of the basic paste without the garcinia decreased as increased storage time. However, the DPPH radical scavenging activity and the FRAP activity of the garcinia Keang-hleung paste with and without salt increased during 2 months of storage period and then decreased as increased storage time. Total viable count (TVC) of all paste samples were in the range of $10^{2}-10^{3} \mathrm{cfu} / \mathrm{g}$. Yeast and mold counts of basic and garcinia Keang-hlueng paste were less than $30 \mathrm{cfu} / \mathrm{g}$ during storage. While, yeast and mold counts of garcinia Keang-hlueng paste without salt were less than $10^{2} \mathrm{cfu} / \mathrm{g}$ during storage. Lactic acid bacteria counts of garcinia Keang-hlueng paste were less than $30 \mathrm{cfu} / \mathrm{g}$ during storage. While, lactic acid bacteria counts of the basic and garcinia Keang-hlueng paste without salt were less than $10^{2} \mathrm{cfu} / \mathrm{g}$ during storage. However, Staphylococcus aureus, Bacilluus cereus, Clostridium perfringens, Salmonella spp., Escherichia coli and coliforms were not detected in all treatments throughout the storage period.
\end{abstract}

Keywords: Keang-hleung Paste; Southern Sour Curry; Garcinia; Antioxidant; Shelf-Life; Thailand

\section{Introduction}

Free radicals are unstable and highly reactive, and energized molecules have unpaired electron such as superoxide, hydroxyl, peroxyl and alkoxyl. Outside the living cell, these compounds are produced by sunlight, ultraviolet light, ionizing radiation, chemical reactions and metabolic processes; however, they are continuously produced in the human body and also controlled by endogenous enzyme (superoxide dismutase, glutathione peroxidase, catalase). An over-production of these species, exposure to external oxidant substance or failure in

\footnotetext{
*Corresponding author.
}

the defense mechanisms, leads to damaging of valuable bio-molecules (DNA, lipids, proteins) which associated with and increased risk of cardiovascular disease, cancer and other chronic disease [1]. In recent years, human health related to nutrition, fitness and beauty has exaggerated concerns over diet. Therefore, a new diet health paradigm is more interesting.

The meaning of some Thai words such as "keang" means curry which is hot and spicy, while "som" means sour and "hleung" means yellow color as pigment derived from turmeric rhizome. Keang-hleung or Southern sour curry soup is now popular not only in southern part of Thailand but also others. It is also claimed as a healthy 
food because of low calories due to less fat but high propotion of vegetable. Southern sour curry normally contains many kinds of vegetables; therefore it has high fiber which is good for health. Moreover, the ingredients used in the paste are turmeric rhizome, garlic, shallot and chili which have been reported as a source of antimicrobial and antioxidant compounds [2-5].

For cooking the sour curry soup, souring agent such as lime juice, tamarind juice, and garcinia fruit, or any aviable sour fruit will be used. Hydroxy citric acid, an active compound found in garcinia fruit, a local fruit of the Southern part of Thailand, can help metabolize glucose and carbohydrates and reduce the accumulation of fat $[6,7]$. Currently, garcinia powder or garcinia extract is used as weight controlling product. Siripongvutikorn et al. [8] reported that using garcinia as souring agent in instant Tom-Yum mixed was more acceptable compared with commercial instant Tom-Yum. Therefore, garcinia Keang-hleung is planned to make for convenient product and may also serve some functional property. However, the addition of garcinia in the paste may alter some qualities, and antioxidant property then consumer acceptability of the paste and the soup were also investigated.

\section{Material and Methods}

\subsection{Material}

Turmeric rhizomes (Curcuma longa), garlic (Allium sativum), dried finger chili (Capsicum annuum), shallot (Allium ascalonicum) and dried garcinia (Garcinia atroviridis) were purchased from a local market in Hat Yai, Thailand.

\subsection{Chemicals}

All chemicals and reagents were of analytical grade. 2, 2-diphenyl-1-picrylhydrazyl (DPPH), 2,4,6-Tripyridyl-striazine (TPTZ), gallic acid, ferric chloride hexahydrate $\left(\begin{array}{lll}\mathrm{FeCl}_{3} & 6 \mathrm{H}_{2} \mathrm{O}\end{array}\right)$ were purchased from Fluka, Sigma Chemical Co. (St. Louis, MQ, USA). Hydrochloric acid, sodium acetate, folin-Ciocalteu reagent, sodium carbonate $\left(\mathrm{Na}_{2} \mathrm{CO}_{3}\right)$, absolute ethanol were purchased from Merck (Darmstadt, Germany).

$$
\begin{aligned}
& \% \text { Total acidity }(\text { citric acid }) \\
& =(\text { vol. of } \mathrm{NaOH} \times \text { Normality of } \mathrm{NaOH} \times 0.064 \times 100) / \text { wt. of sample }[10] .
\end{aligned}
$$

\subsection{Analyses}

\subsubsection{Determination of Total Phenolic Compound Content}

Total phenolic content of the extracted sample was determined using the Folin-Ciocalteu assay reported by

\subsection{Keang-hleang Paste Preparation}

All spices were sorted, trimmed and washed thoroughly to remove dust and dirt, then soaked in $150 \mathrm{ppm}$ and 10 ppm of chlorine solution, respectively for 1 minute, then weighed according to basic recipe before added $20 \%$ salt then divided in to 3 groups as P1, P2 and P3. Based on calculation the compositions of each paste were differences as showed in Table 1. All mentioned ingredients were then ground with blender (Moulinex, TYPE 276, France) to make a fine paste (20 - 40 mesh).

\subsection{Extraction Procedure}

The paste $100 \mathrm{~g}$ (fresh weight) was extracted with $300 \mathrm{ml}$ of distilled water then stirred with a magnetic stirrer 12 $\mathrm{hr}$ before being subjected to filter through cheesecloth and centrifuge at $2000 \mathrm{~g}$ for $25 \mathrm{~min}$. Thereafter, the supernatant was dried with freezed dryer and kept at $-20^{\circ} \mathrm{C}$ until used.

\subsection{Physical Qualities}

\subsection{1. pH Values}

Ten $\mathrm{g}$ of the paste was homogenized in $40 \mathrm{ml}$ distilled water for $1 \mathrm{~min}$ with the homogenizer (Wiggen Hauser D500, Germany) and measured for $\mathrm{pH}$ at room temperature with a Satorius Docu-pH Meter (Germany) [9].

\subsubsection{Color Values}

Color values of the extracts were measured using a color meter (Hunter Lab Universal Software). The color values were express as CIE Lab* coordinates where $L^{*}$ represents the luminosity $(0=$ black; $100=$ white $), a^{*}$ the redness $\left(a^{*}>0\right)$ or $\left(a^{*}<0\right)$ and $b^{*}$ the blueness $\left(b^{*}>0\right)$ or yellowness $\left(b^{*}<0\right)$.

\subsection{Chemical Qualities}

\section{Titratable Acidity}

The $5 \mathrm{ml}$ of sample was diluted with $45 \mathrm{ml}$ distilled water and titrated with $0.1 \mathrm{~N}$ sodium hydroxide to reach $\mathrm{pH}$ 8.1. The results were expressed as percentage of citric acid ( $\mathrm{g}$ citric acid/100 $\mathrm{g}$ ) as equation below.
Kahkonen et al. [11] with some modification. Briefly, the extracted sample $(20 \mu \mathrm{l})$ was introduced into 96 well plates, followed by $100 \mu \mathrm{l}$ of Folin-Ciocalteu's reagent and $80 \mu \mathrm{l}$ of sodium carbonate $(7.5 \% \mathrm{w} / \mathrm{v})$. The plates were shaken vigorously and left at ambient temperature $\left(29^{\circ} \mathrm{C} \pm 2^{\circ} \mathrm{C}\right)$ for $30 \mathrm{~min}$ in the dark. Then the absorbance 
Table 1. The ingredient compositions in any paste formula.

\begin{tabular}{cccc}
\hline \multirow{2}{*}{ Component } & \multicolumn{3}{c}{ Formulation (\%) } \\
\cline { 2 - 4 } & P1 & P2 & P3 \\
\hline Garlic & 10 & 8 & 10.5 \\
Shallot & 30 & 24.5 & 32 \\
Chilli & 30 & 24.5 & 32 \\
Turmeric Rhizome & 10 & 8 & 10.5 \\
Garcinia & - & 15 & 15 \\
Salt & 20 & 20 & - \\
\hline
\end{tabular}

P1: Basic Keang-hleung paste. P2: Garcinia Keang-hleung paste with 20\% salt. P3: Garcinia Keang-hleung paste without $20 \%$ salt.

was measured at $765 \mathrm{~nm}$ using the microplate reader (PowerWare X, Biotek, USA). Gallic acid was used as antioxidant standard, and reported as gallic/100g sample of dry weight.

\subsubsection{Determination of Antioxidant Activity \\ 1) DPPH Scavenging Activity}

DPPH scavenging activity was described by $\mathrm{Wu}$ et al. [12] with some modification. Briefly, a $100 \mu \mathrm{l}$ of each sample was mixed with $100 \mu$ of $0.3 \mathrm{mM}$ DPPH dissolved in $75 \%$ ethanol. The mixture was shaken vigorously and left at ambient temperature for $30 \mathrm{~min}$ in the dark. The DPPH scavenging activity was determined by measuring the absorbance at $517 \mathrm{~nm}$ using the microplate reader (PowerWare X, Biotek, USA).

\section{2) FRAP Antioxidant Activity}

The FRAP assay was done according to Benzie and Strain [13] with some modifications. The stock solutions included $300 \mathrm{mM}$ acetate buffer $\left(3.1 \mathrm{~g} \mathrm{C}_{2} \mathrm{H}_{3} \mathrm{NaO}_{2} \cdot 3 \mathrm{H}_{2} \mathrm{O}\right.$ and $\left.16 \mathrm{ml} \mathrm{C}_{2} \mathrm{H}_{4} \mathrm{O}_{2}\right)$, pH 3.6, $10 \mathrm{mM}$ TPTZ $(2,4,6-$ Tripyridyl-s-triazine) solution in $40 \mathrm{mM} \mathrm{HCl}$ and $20 \mathrm{mM}$ $\mathrm{FeCl}_{3} \cdot 6 \mathrm{H}_{2} \mathrm{O}$ solution. The fresh working solution was prepared by mixing $25 \mathrm{ml}$ acetate buffer, $2.5 \mathrm{ml} \mathrm{TPTZ}$ solution and $2.5 \mathrm{ml} \mathrm{FeCl}_{3} \cdot 6 \mathrm{H}_{2} \mathrm{O}$ solution and then warmed at $37^{\circ} \mathrm{C}$ before used. The extracted sample $(15 \mu \mathrm{l})$ was allowed to react with $285 \mu \mathrm{l}$ of the FRAP solution for 30 min in the dark condition. Reading of the colored products [ferrous tripyridyltriazine complex] was then taken at $593 \mathrm{~nm}$ using the microplate reader (PowerWare X, Biotek, USA).

\subsection{Microbiological Analyses}

Total viable count (mesophilic bacteria), Coliforms, Escherichia coli, Staphylococcus aureus, Bacillus cereus, Clostridium perfringens, Salmonella and Lactic acid bacteria as well as yeast and mold were followed BAM, [14]. Briefly, Twenty-five grams of the paste was blended with $225 \mathrm{ml}$ of $0.1 \%$ peptone water. Serial dilu- tion was made at $10^{-1}$ to $10^{-6}$ by using the $0.1 \%$ peptone water. Appropriate dilution was determined as mentioned in BAM [14].

\subsection{Statistical Analysis}

Data were subjected to analysis of variance, and mean comparison were made using Duncan's new multiple range test. Statistical analyses were carried out using the SPSS statistical software version 6 (SPSS, Inc., Chicago, IL)

\section{Results and Discussion}

\subsection{Quality Changes of Keang-Hleung Paste During Storage at Ambient Temperature $\left(29^{\circ} \mathrm{C} \pm 2^{\circ} \mathrm{C}\right)$ and $4^{\circ} \mathrm{C} \pm 2^{\circ} \mathrm{C}$}

\section{Physical and Chemical Properties}

The color value interm of $L^{*}, a^{*}$ and $b^{*}$ of the basic and garcinia Keang-hleung with and without $20 \%$ salt then stored at ambient temperature and $4^{\circ} \mathrm{C}$ were presented in Tables 2 and 3, respectively. A decreased of color values particularly $a^{*}$ value, redness, during storage may due to

Table 2. Color values of basic and garcinia Keang-hleung paste during storage at ambient temperature.

\begin{tabular}{|c|c|c|c|c|}
\hline \multirow[t]{2}{*}{ Treatment } & \multirow{2}{*}{$\begin{array}{l}\text { Storage } \\
\text { (month) }\end{array}$} & \multicolumn{3}{|c|}{ Color } \\
\hline & & $L^{*}$ & $a^{*}$ & $b^{*}$ \\
\hline \multirow{7}{*}{$\begin{array}{l}\text { Basic Keang- } \\
\text { hleung Paste }\end{array}$} & 0 & $29.82 \pm 0.01^{\mathrm{bc}}$ & $36.44 \pm 0.09^{\mathrm{a}}$ & $45.76 \pm 0.10^{c}$ \\
\hline & 1 & $29.27 \pm 0.01^{b c}$ & $34.96 \pm 0.03^{b}$ & $44.32 \pm 0.07^{\mathrm{cd}}$ \\
\hline & 2 & $29.22 \pm 0.04^{b c}$ & $32.82 \pm 0.02^{\mathrm{bc}}$ & $44.41 \pm 0.33^{\mathrm{cd}}$ \\
\hline & 3 & $28.01 \pm 0.12^{\mathrm{c}}$ & $32.88 \pm 0.26^{\mathrm{bc}}$ & $44.98 \pm 0.33^{\mathrm{cd}}$ \\
\hline & 4 & $28.16 \pm 0.03^{c}$ & $30.87 \pm 0.06^{\mathrm{c}}$ & $44.55 \pm 0.07^{\mathrm{cd}}$ \\
\hline & 5 & $28.39 \pm 0.20^{\mathrm{c}}$ & $30.38 \pm 0.17^{\mathrm{c}}$ & $43.16 \pm 0.06^{\mathrm{cd}}$ \\
\hline & 6 & $25.86 \pm 0.06^{\mathrm{e}}$ & $30.59 \pm 0.07^{\mathrm{c}}$ & $43.04 \pm 0.14^{\mathrm{cd}}$ \\
\hline \multirow{7}{*}{$\begin{array}{c}\text { Garcinia } \\
\text { Keang-hleung } \\
\text { Paste with Salt } \\
20 \%\end{array}$} & 0 & $28.72 \pm 0.13^{\mathrm{c}}$ & $34.18 \pm 0.20^{\mathrm{b}}$ & $47.66 \pm 0.05^{\mathrm{b}}$ \\
\hline & 1 & $28.14 \pm 0.01^{\mathrm{c}}$ & $32.21 \pm 0.02^{\mathrm{bc}}$ & $47.30 \pm 0.22^{b}$ \\
\hline & 2 & $28.97 \pm 0.11^{\mathrm{c}}$ & $34.11 \pm 0.05^{\mathrm{b}}$ & $45.14 \pm 0.21^{\mathrm{c}}$ \\
\hline & 3 & $28.44 \pm 0.07^{\mathrm{c}}$ & $30.87 \pm 0.12^{c}$ & $45.64 \pm 0.25^{\mathrm{c}}$ \\
\hline & 4 & $27.63 \pm 0.03^{d}$ & $31.82 \pm 0.04^{\mathrm{c}}$ & $45.59 \pm 0.04^{\mathrm{c}}$ \\
\hline & 5 & $26.56 \pm 0.06^{\mathrm{d}}$ & $31.13 \pm 0.11^{\mathrm{c}}$ & $43.12 \pm 0.44^{\mathrm{cd}}$ \\
\hline & 6 & $26.53 \pm 0.51^{\mathrm{d}}$ & $30.09 \pm 0.19^{c}$ & $43.33 \pm 0.26^{\mathrm{cd}}$ \\
\hline \multirow{7}{*}{$\begin{array}{c}\text { Garcinia } \\
\text { Keang-hleung } \\
\text { Paste without } \\
\text { Salt } 20 \%\end{array}$} & 0 & $31.14 \pm 0.02^{\mathrm{a}}$ & $34.22 \pm 0.06^{b c}$ & $48.99 \pm 0.14^{\mathrm{a}}$ \\
\hline & 1 & $31.23 \pm 0.03^{\mathrm{a}}$ & $33.82 \pm 0.06^{\mathrm{bc}}$ & $48.68 \pm 0.05^{\mathrm{a}}$ \\
\hline & 2 & $30.81 \pm 0.02^{\mathrm{ab}}$ & $33.00 \pm 0.06^{\mathrm{bc}}$ & $48.75 \pm 0.26^{\mathrm{a}}$ \\
\hline & 3 & $29.35 \pm 0.03^{b c}$ & $32.87 \pm 0.02^{\mathrm{c}}$ & $48.50 \pm 0.28^{a}$ \\
\hline & 4 & $28.75 \pm 0.06^{\mathrm{c}}$ & $30.46 \pm 0.07^{\mathrm{c}}$ & $48.71 \pm 0.20^{\mathrm{a}}$ \\
\hline & 5 & $28.33 \pm 0.08^{\mathrm{c}}$ & $30.31 \pm 0.16^{\mathrm{c}}$ & $48.66 \pm 0.07^{\mathrm{a}}$ \\
\hline & 6 & $28.29 \pm 0.14^{\mathrm{c}}$ & $30.69 \pm 0.15^{\mathrm{c}}$ & $46.69 \pm 0.15^{\mathrm{bc}}$ \\
\hline
\end{tabular}

Each value is expressed as a mean $\pm \mathrm{SD}(\mathrm{n}=3)$; a - e mean that with differ ent letters within a column are significant different $(\mathrm{p}<0.05)$ 
Table 3. Color values of basic and garcinia Keang-hleung paste during storage at $4^{\circ} \mathrm{C} \pm 2^{\circ} \mathrm{C}$.

\begin{tabular}{|c|c|c|c|c|}
\hline \multirow{2}{*}{ Treatment } & \multirow{2}{*}{$\begin{array}{l}\text { Storage } \\
\text { (month) }\end{array}$} & \multicolumn{3}{|c|}{ Color } \\
\hline & & $L^{*}$ & $a^{*}$ & $b^{*}$ \\
\hline \multirow{7}{*}{$\begin{array}{l}\text { Basic Keang- } \\
\text { hleung Paste }\end{array}$} & 0 & $29.82 \pm 0.01^{\mathrm{a}}$ & $36.44 \pm 0.09^{\mathrm{a}}$ & $45.76 \pm 0.10^{b}$ \\
\hline & 1 & $29.53 \pm 0.01^{\mathrm{a}}$ & $34.11 \pm 0.01^{b}$ & $44.89 \pm 0.16^{b c}$ \\
\hline & 2 & $29.55 \pm 0.05^{\mathrm{a}}$ & $34.64 \pm 0.07^{\mathrm{b}}$ & $44.50 \pm 0.38^{b c}$ \\
\hline & 3 & $28.84 \pm 0.07^{\mathrm{ab}}$ & $34.04 \pm 0.16^{\mathrm{b}}$ & $44.88 \pm 0.14^{b c}$ \\
\hline & 4 & $28.83 \pm 0.04^{\mathrm{ab}}$ & $34.53 \pm 0.06^{\mathrm{b}}$ & $44.10 \pm 0.08^{b c}$ \\
\hline & 5 & $28.66 \pm 0.23^{\mathrm{ab}}$ & $31.20 \pm 0.10^{\mathrm{d}}$ & $44.66 \pm 0.06^{\mathrm{bc}}$ \\
\hline & 6 & $25.90 \pm 0.05^{\mathrm{d}}$ & $30.56 \pm 0.09^{d}$ & $43.60 \pm 0.10^{\mathrm{c}}$ \\
\hline \multirow{7}{*}{$\begin{array}{c}\text { Garcinia } \\
\text { Keang-hleung } \\
\text { Paste with Salt } \\
20 \%\end{array}$} & 0 & $28.72 \pm 0.13^{\mathrm{ab}}$ & $34.18 \pm 0.20^{\mathrm{b}}$ & $47.66 \pm 0.05^{b}$ \\
\hline & 1 & $27.86 \pm 0.05^{\mathrm{bc}}$ & $29.05 \pm 0.11^{\mathrm{e}}$ & $45.99 \pm 0.38^{b c}$ \\
\hline & 2 & $27.52 \pm 0.03^{b c}$ & $29.51 \pm 0.10^{\mathrm{e}}$ & $45.07 \pm 0.25^{\mathrm{bc}}$ \\
\hline & 3 & $26.88 \pm 0.04^{c}$ & $29.46 \pm 0.04^{\mathrm{e}}$ & $46.54 \pm 0.24^{b}$ \\
\hline & 4 & $27.80 \pm 0.02^{\mathrm{bc}}$ & $30.15 \pm 0.05^{\mathrm{de}}$ & $46.31 \pm 0.19^{b}$ \\
\hline & 5 & $27.18 \pm 0.02^{\mathrm{bc}}$ & $26.22 \pm 0.06^{\mathrm{f}}$ & $46.99 \pm 0.14^{b}$ \\
\hline & 6 & $27.90 \pm 0.03^{b c}$ & $26.13 \pm 0.13^{f}$ & $46.17 \pm 0.07^{b}$ \\
\hline \multirow{7}{*}{$\begin{array}{c}\text { Garcinia } \\
\text { Keang-hleung } \\
\text { Paste without } \\
\text { Salt } 20 \%\end{array}$} & 0 & $31.14 \pm 0.02^{\mathrm{a}}$ & $34.22 \pm 0.06^{\mathrm{b}}$ & $48.99 \pm 0.14^{\mathrm{a}}$ \\
\hline & 1 & $30.92 \pm 0.05^{\mathrm{a}}$ & $32.03 \pm 0.19^{c}$ & $48.82 \pm 0.18^{\mathrm{a}}$ \\
\hline & 2 & $30.80 \pm 0.01^{\mathrm{a}}$ & $30.15 \pm 0.08^{\mathrm{de}}$ & $48.96 \pm 0.26^{\mathrm{a}}$ \\
\hline & 3 & $29.42 \pm 0.01^{\mathrm{a}}$ & $30.38 \pm 0.26^{\mathrm{de}}$ & $48.46 \pm 0.20^{\mathrm{a}}$ \\
\hline & 4 & $28.47 \pm 0.03^{\mathrm{ab}}$ & $30.73 \pm 0.07^{\mathrm{de}}$ & $48.15 \pm 0.11^{\mathrm{a}}$ \\
\hline & 5 & $28.35 \pm 0.02^{\mathrm{ab}}$ & $27.03 \pm 0.03^{\mathrm{f}}$ & $47.40 \pm 0.08^{\mathrm{ab}}$ \\
\hline & 6 & $28.22 \pm 0.02^{\mathrm{ab}}$ & $27.59 \pm 0.07^{\mathrm{f}}$ & $47.50 \pm 0.40^{\mathrm{a}}$ \\
\hline
\end{tabular}

Each value is expressed as a mean $\pm \mathrm{SD}(\mathrm{n}=3)$; a - $\mathrm{f}$ mean that with different letters within a column are significant different $(\mathrm{p}<0.05)$.

degradation of carotenoids oxidation process mainly $\beta$-carotene during storage period $[15,16]$. Similar result was found in study of Ketsa and Pangkoolm [17,18] who reported that the fading of durian pulp color was most probably due to degradation of $\beta$-carotene because the curcuminoids were readily decomposed when exposed to bright light [19]. Moreover, Coneellon et al. [20] addressed that oxidized products such as mono-, di-phenol and quinones of phenolic compounds were unstable and rapidly react with amino acid or protein particularly at $30^{\circ} \mathrm{C}$, generating brown pigments by polymerization. However, kepting the paste in lower temperature, $4^{\circ} \mathrm{C}$, retained more color values particularly $b^{*}$ value. It pointed out that enzymatic oxidation of natural phenolic compounds was partly inhibition. The difference of $a^{*}$ value between the basic paste and garcinia paste with and without salt was noticed. The basic paste had more $a^{*}$ value compared with the garcinia paste may due to $\beta$-carotene bleaching affect [21] as function of hydroxyl citric acid derived from garcinia leading to redness reducing and yellowness increasing.

The $\mathrm{pH}$ values and acidity $(\mathrm{g} / 100 \mathrm{~g})$ during storage time of the Keang-hleung paste were presented in Tables 4 and 5 , respectively. $\mathrm{pH}$ values and acidity of basic Keanghleung paste kept at ambient temperature and $4^{\circ} \mathrm{C} \pm 2^{\circ} \mathrm{C}$ tended to decrease and increase, respectively during storage. This may due to growth of lactic acid bacteria producing lactic acid or acetic acid [22,23]. As expected, the lower of $\mathrm{pH}$ and higher of acidity $(\mathrm{g} / 100 \mathrm{~g})$ were found in the paste added with garcinia may due to organic acid mainly hydroxy citric acid containing in garcinia fruit.

The $A_{w}$ of Keang-hleung paste during storage at ambient temperature and $4^{\circ} \mathrm{C} \pm 2^{\circ} \mathrm{C}$ were presented in Tables 4 and 5 , respectively. The result showed that $A_{w}$ did not

Table 4. pH values, acidity (g/100g) change and $A_{w}$ of Keanghleung paste during storage at ambient temperature.

\begin{tabular}{|c|c|c|c|c|}
\hline Treatment & $\begin{array}{c}\text { Storage } \\
\text { (months) }\end{array}$ & $\mathrm{pH}$ & $\begin{array}{l}\text { Acidity } \\
(\mathrm{g} / 100 \mathrm{~g})\end{array}$ & $\mathrm{A}_{\mathrm{w}}$ \\
\hline \multirow{7}{*}{$\begin{array}{l}\text { Basic Keang- } \\
\text { hleung Paste }\end{array}$} & 0 & $5.08 \pm 0.01^{\mathrm{a}}$ & $0.39 \pm 0.01^{\mathrm{c}}$ & $0.79 \pm 0.001^{b}$ \\
\hline & 1 & $5.04 \pm 0.01^{\mathrm{a}}$ & $0.44 \pm 0.01^{\mathrm{c}}$ & $0.80 \pm 0.001^{b}$ \\
\hline & 2 & $4.99 \pm 0.01^{\mathrm{a}}$ & $0.44 \pm 0.00^{\mathrm{c}}$ & $0.79 \pm 0.001^{b}$ \\
\hline & 3 & $4.98 \pm 0.02^{\mathrm{a}}$ & $0.44 \pm 0.02^{\mathrm{c}}$ & $0.80 \pm 0.001^{b}$ \\
\hline & 4 & $4.98 \pm 0.01^{\mathrm{a}}$ & $0.46 \pm 0.03^{\mathrm{c}}$ & $0.79 \pm 0.001^{b}$ \\
\hline & 5 & $4.91 \pm 0.01^{\mathrm{a}}$ & $0.48 \pm 0.03^{\mathrm{c}}$ & $0.80 \pm 0.001^{b}$ \\
\hline & 6 & $4.85 \pm 0.03^{\mathrm{a}}$ & $0.48 \pm 0.00^{\mathrm{c}}$ & $0.79 \pm 0.001^{\mathrm{b}}$ \\
\hline \multirow{7}{*}{$\begin{array}{c}\text { Garcinia } \\
\text { Keang-hleung } \\
\text { Paste with Salt } \\
20 \%\end{array}$} & 0 & $3.38 \pm 0.02^{\mathrm{c}}$ & $1.41 \pm 0.03^{b}$ & $0.81 \pm 0.001^{\mathrm{b}}$ \\
\hline & 1 & $3.30 \pm 0.00^{c}$ & $1.40 \pm 0.02^{\mathrm{b}}$ & $0.81 \pm 0.001^{\mathrm{b}}$ \\
\hline & 2 & $3.29 \pm 0.01^{\mathrm{c}}$ & $1.43 \pm 0.01^{b}$ & $0.83 \pm 0.001^{\mathrm{b}}$ \\
\hline & 3 & $3.28 \pm 0.01^{\mathrm{c}}$ & $1.50 \pm 0.02^{\mathrm{b}}$ & $0.83 \pm 0.001^{b}$ \\
\hline & 4 & $3.26 \pm 0.01^{\mathrm{c}}$ & $1.56 \pm 0.04^{\mathrm{b}}$ & $0.82 \pm 0.001^{\mathrm{b}}$ \\
\hline & 5 & $3.26 \pm 0.01^{\mathrm{c}}$ & $1.56 \pm 0.03^{\mathrm{b}}$ & $0.82 \pm 0.001^{b}$ \\
\hline & 6 & $3.18 \pm 0.00^{\mathrm{c}}$ & $1.43 \pm 0.01^{\mathrm{b}}$ & $0.83 \pm 0.001^{b}$ \\
\hline \multirow{7}{*}{$\begin{array}{c}\text { Garcinia } \\
\text { Keang-hleung } \\
\text { Paste without } \\
\text { Salt } 20 \%\end{array}$} & 0 & $3.62 \pm 0.01^{\mathrm{b}}$ & $1.59 \pm 0.04^{\mathrm{b}}$ & $0.98 \pm 0.001^{\mathrm{a}}$ \\
\hline & 1 & $3.63 \pm 0.01^{\mathrm{b}}$ & $1.78 \pm 0.03^{\mathrm{ab}}$ & $0.97 \pm 0.001^{\mathrm{a}}$ \\
\hline & 2 & $3.55 \pm 0.01^{\mathrm{b}}$ & $1.85 \pm 0.01^{\mathrm{a}}$ & $0.98 \pm 0.001^{\mathrm{a}}$ \\
\hline & 3 & $3.55 \pm 0.01^{\mathrm{b}}$ & $1.82 \pm 0.03^{\mathrm{a}}$ & $0.97 \pm 0.001^{\mathrm{a}}$ \\
\hline & 4 & $3.54 \pm 0.01^{\mathrm{b}}$ & $1.81 \pm 0.03^{\mathrm{a}}$ & $0.97 \pm 0.001^{\mathrm{a}}$ \\
\hline & 5 & $3.51 \pm 0.01^{\mathrm{b}}$ & $1.95 \pm 0.05^{\mathrm{a}}$ & $0.97 \pm 0.001^{\mathrm{a}}$ \\
\hline & 6 & $3.45 \pm 0.01^{\mathrm{b}}$ & $1.99 \pm 0.05^{\mathrm{a}}$ & $0.98 \pm 0.001^{\mathrm{a}}$ \\
\hline
\end{tabular}

Each value is expressed as a mean $\pm \mathrm{SD}(\mathrm{n}=3)$; a - c mean that with different letters within a column are significant different $(\mathrm{p}<0.05)$. 
Table 5. pH values, acidity (g/100 g) and $A_{w}$ of Keang-hleung paste during storage at $4^{\circ} \mathrm{C} \pm 2^{\circ} \mathrm{C}$.

\begin{tabular}{|c|c|c|c|c|}
\hline Treatment & $\begin{array}{l}\text { Storage } \\
\text { (month) }\end{array}$ & $\mathrm{pH}$ & $\begin{array}{l}\text { Acidity } \\
(\mathrm{g} / 100 \mathrm{~g})\end{array}$ & $\mathrm{A}_{\mathrm{w}}$ \\
\hline \multirow{7}{*}{$\begin{array}{l}\text { Basic Keang- } \\
\text { hleung Paste }\end{array}$} & 0 & $5.08 \pm 0.01^{\mathrm{a}}$ & $0.39 \pm 0.01^{\mathrm{c}}$ & $0.79 \pm 0.001^{b}$ \\
\hline & 1 & $5.03 \pm 0.00^{\mathrm{a}}$ & $0.44 \pm 0.01^{\mathrm{c}}$ & $0.79 \pm 0.001^{\mathrm{b}}$ \\
\hline & 2 & $4.94 \pm 0.01^{\mathrm{a}}$ & $0.44 \pm 0.01^{\mathrm{c}}$ & $0.79 \pm 0.001^{\mathrm{b}}$ \\
\hline & 3 & $4.93 \pm 0.02^{\mathrm{a}}$ & $0.43 \pm 0.00^{c}$ & $0.80 \pm 0.001^{\mathrm{b}}$ \\
\hline & 4 & $4.95 \pm 0.01^{\mathrm{a}}$ & $0.43 \pm 0.01^{\mathrm{c}}$ & $0.79 \pm 0.001^{\mathrm{b}}$ \\
\hline & 5 & $4.85 \pm 0.01^{\mathrm{a}}$ & $0.45 \pm 0.00^{\mathrm{c}}$ & $0.79 \pm 0.001^{b}$ \\
\hline & 6 & $4.84 \pm 0.03^{\mathrm{a}}$ & $0.46 \pm 0.01^{\mathrm{c}}$ & $0.79 \pm 0.001^{b}$ \\
\hline \multirow{7}{*}{$\begin{array}{c}\text { Garcinia } \\
\text { Keang-hleung } \\
\text { Paste with Salt } \\
20 \%\end{array}$} & 0 & $3.38 \pm 0.02^{\mathrm{c}}$ & $1.41 \pm 0.03^{b}$ & $0.81 \pm 0.001^{b}$ \\
\hline & 1 & $3.32 \pm 0.00^{\mathrm{c}}$ & $1.40 \pm 0.02^{\mathrm{b}}$ & $0.82 \pm 0.001^{\mathrm{b}}$ \\
\hline & 2 & $3.28 \pm 0.01^{\mathrm{c}}$ & $1.43 \pm 0.03^{\mathrm{b}}$ & $0.81 \pm 0.001^{b}$ \\
\hline & 3 & $3.25 \pm 0.01^{\mathrm{c}}$ & $1.50 \pm 0.01^{b}$ & $0.82 \pm 0.001^{\mathrm{b}}$ \\
\hline & 4 & $3.24 \pm 0.02^{\mathrm{c}}$ & $1.56 \pm 0.04^{\mathrm{b}}$ & $0.82 \pm 0.001^{\mathrm{b}}$ \\
\hline & 5 & $3.19 \pm 0.01^{\mathrm{c}}$ & $1.56 \pm 0.03^{b}$ & $0.82 \pm 0.001^{b}$ \\
\hline & 6 & $3.20 \pm 0.01^{\mathrm{c}}$ & $1.43 \pm 0.02^{\mathrm{b}}$ & $0.81 \pm 0.001^{b}$ \\
\hline \multirow{7}{*}{$\begin{array}{c}\text { Garcinia } \\
\text { Keang-hleung } \\
\text { Paste } \\
\text { without Salt } \\
20 \%\end{array}$} & 0 & $3.62 \pm 0.01^{b}$ & $1.59 \pm 0.04^{b}$ & $0.98 \pm 0.001^{\mathrm{a}}$ \\
\hline & 1 & $3.79 \pm 0.01^{\mathrm{b}}$ & $1.78 \pm 0.05^{\mathrm{ab}}$ & $0.98 \pm 0.001^{\mathrm{a}}$ \\
\hline & 2 & $3.71 \pm 0.02^{\mathrm{b}}$ & $1.85 \pm 0.04^{\mathrm{a}}$ & $0.98 \pm 0.001^{\mathrm{a}}$ \\
\hline & 3 & $3.66 \pm 0.01^{\mathrm{b}}$ & $1.82 \pm 0.07^{\mathrm{a}}$ & $0.98 \pm 0.001^{\mathrm{a}}$ \\
\hline & 4 & $3.53 \pm 0.04^{b}$ & $1.81 \pm 0.04^{\mathrm{a}}$ & $0.98 \pm 0.001^{\mathrm{a}}$ \\
\hline & 5 & $3.52 \pm 0.01^{\mathrm{b}}$ & $1.95 \pm 0.05^{\mathrm{a}}$ & $0.98 \pm 0.001^{\mathrm{a}}$ \\
\hline & 6 & $3.50 \pm 0.01^{\mathrm{b}}$ & $1.99 \pm 0.06^{\mathrm{a}}$ & $0.98 \pm 0.001^{\mathrm{a}}$ \\
\hline
\end{tabular}

Each value is expressed as a mean $\pm \mathrm{SD}(\mathrm{n}=3)$ a - c means that with different letters within a column are significant different $(\mathrm{p}<0.05)$.

change when during storage increased due to property of packaging (LLDPE/Nylon) which protected moisture permeability and oxygen. The lowest $\mathrm{A}_{\mathrm{w}}$ was found in the basic paste while added garcinia in the paste particularly without salt yielded more $A_{w}$. This evident pointed out that washing step reabsorbed water as free water resulting to higher $\mathrm{A}_{\mathrm{w}}$. It is the reason why draining step needs to be property applied otherwise shorten shelf-life would be occur afterward. In addition, it confirmed that certain salt content could improve shelf-life by reducing $\mathrm{A}_{\mathrm{w}}$. As well known food products having $\mathrm{A}_{\mathrm{w}}$ less than 0.85 are quite safe for pathogenic or spoilage bacteria and have more shelf-life compared with another product having $A_{w}$ higher than 0.85 [24].

The results also showed that storage temperature did not have any effect on $\mathrm{pH}, \%$ acidity and $\mathrm{A}_{\mathrm{w}}$. The major factors controlling these mentioned values depended on the added ingredients particularly garcinia and salt.

\subsection{Total Phenolic Content and Antioxidant of Keang-hleung Paste with and without Garcinia}

Total phenolic contents of the pastes during storage at 29 \pm 2 and $4{ }^{\circ} \mathrm{C} \pm 2^{\circ} \mathrm{C}$ were presented in Figures 1 and 2, respectively. Total phenolic content in the garcinia paste without added salt was highest compared with other pastes may due to highest of spices used. However, at the end of storage (6 mo), total phenolic content of each paste was quite similar meant that faster degradation of phenolic compounds in the garcinia paste without added salt occurred. It pointed out that salt concentration was major role to inhibit enzymatic reaction derived from spice materials. Surprising, total phenolic content in the garcinia paste with added salt kept at ambient temperature was lowest after storage for 2 mo (Figure 1) meant that both salt and garcinia may destroy or bleach some phenolic compounds. However, the basic paste kept at $4^{\circ} \mathrm{C} \pm 2^{\circ} \mathrm{C}$ (Figure 2) seemed to be lowest compared with others even at end of storage ( $6 \mathrm{mo})$ was not different.

\subsection{Antioxidant Activities}

\subsubsection{DPPH Free Radical Scavenging}

$\mathrm{DPPH}^{*}$ is a free radical compound that has been widely

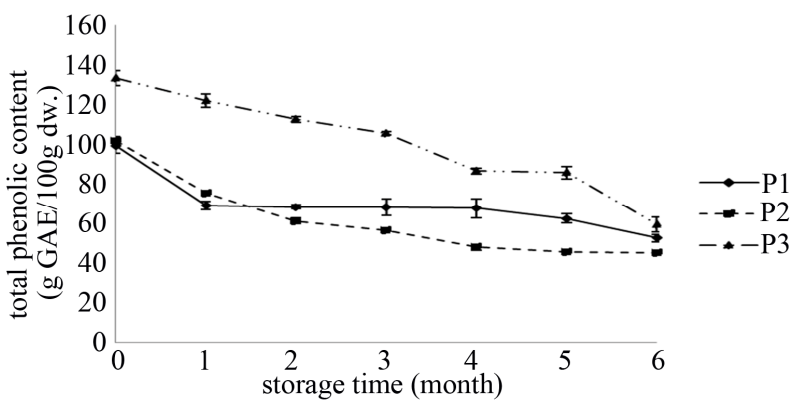

Figure 1. Total phenolic contents of the Keang-hleung paste during storage at $29^{\circ} \mathrm{C} \pm 2^{\circ} \mathrm{C}$. P1: Basic Keang-hleung paste. P2: Garcinia Keang-hleung paste with $20 \%$ salt. P3: Garcinia Keang-hleung paste without $20 \%$ salt.

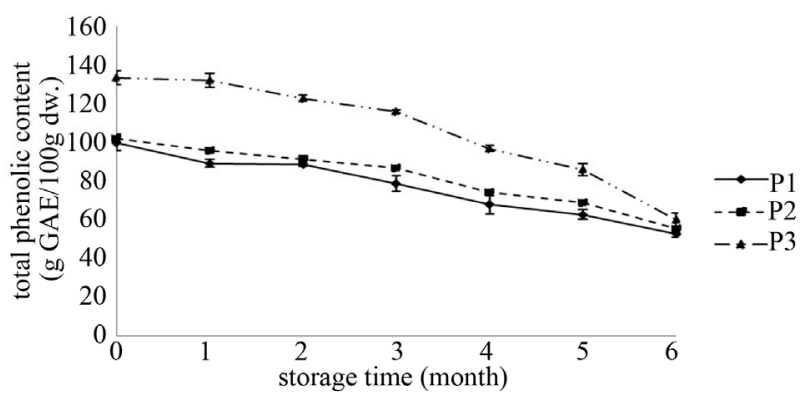

Figure 2. Total phenolic contents of the Keang-hleung paste during storage at $4^{\circ} \mathrm{C} \pm 2^{\circ} \mathrm{C}$. P1: Basic Keang-hleung paste. P2: Garcinia Keang-hleung paste with $20 \%$ salt. P3: Garcinia Keang-hleung paste without $20 \%$ salt. 
used to determine the free radical scavenging capacity of various samples $[25,26]$ because of its stability (in radical form), simplicity and fast assay [27]. The DPPH radical scavenging activities of the pastes during storage at $29 \pm$ 2 and $4^{\circ} \mathrm{C} \pm 2{ }^{\circ} \mathrm{C}$ were presented in Figures 3 and 4, respectively. The results showed that the $\mathrm{DPPH}^{\circ}$ radical scavenging activity of the basic paste kept at ambient temperature and $4^{\circ} \mathrm{C}$ decreased as storage time increased. Surprising again, initial DPPH radical scavenging activity was highest in the basic paste even low in total phenolic content. This result confirmed that antioxidant activity may not well relate to total phenolic content [28]. DPPH radical scavenging activity of the garcinia paste without added salt kept in both storage temperatures increased in the first 2 mo before decreased and was lowest at end of storage. An increase of the activity may cause by polyphenol oxidase [20] at the first period of time yielding some active compounds however, without salt and high temperature may allow microbial growth as show in Table 6. It meant that degradated compounds derived from the paste were low in $\mathrm{H}^{+}$donor ability. A decrease of DPPH radical scavenging activity well relates to a decrease of total phenolic compounds as mentioned before.

\subsubsection{Ferric Reducing/Antioxidant Power (FRAP)}

The FRAP activity of the basic paste kept in both tem-

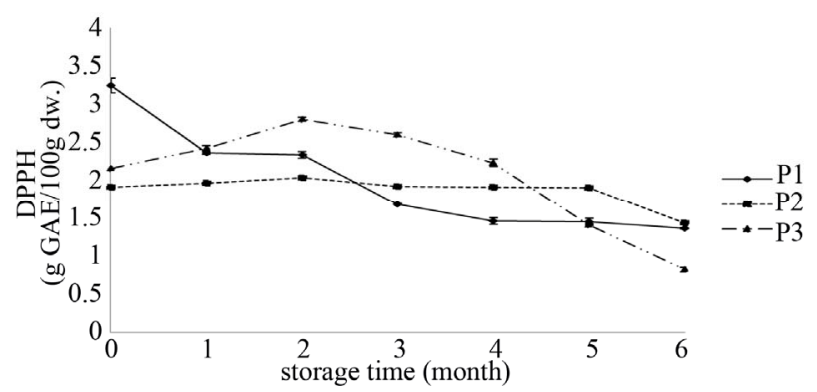

Figure 3. The DPPH scavenging activity of Keang-hleung paste during storage at $29^{\circ} \mathrm{C} \pm 2^{\circ} \mathrm{C}$. P1: Basic Keang-hleung paste. P2: Garcinia Keang-hleung paste with $20 \%$ salt. P3: Garcinia Keang-hleung paste without $20 \%$ salt.

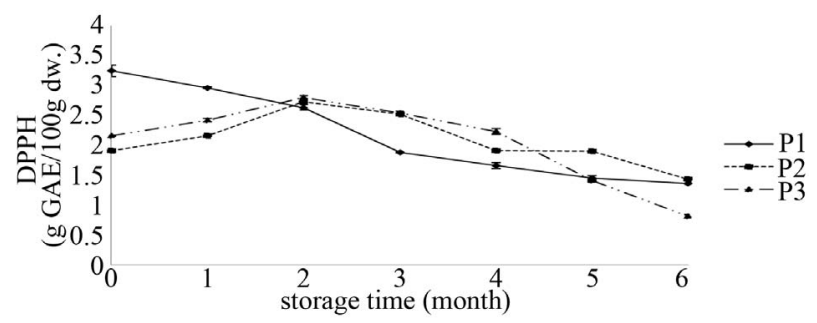

Figure 4. The DPPH scavenging activity of Keang-hleung paste during storage at $4^{\circ} \mathrm{C} \pm 2^{\circ} \mathrm{C}$. P1: Basic Keang-hleung paste. P2: Garcinia Keang-hleung paste with $20 \%$ salt. P3: Garcinia Keang-hleung paste without $20 \%$ salt.
Table 6. Microbiological quality in basic and garcinia Keang-hlueng paste with and without salt during storage at ambient temperature and $4^{\circ} \mathrm{C} \pm 2^{\circ} \mathrm{C}$.

\begin{tabular}{|c|c|c|c|c|}
\hline \multirow{2}{*}{ Treatment } & \multicolumn{4}{|c|}{ Bacteria Count (cfu/g) } \\
\hline & $\begin{array}{c}\text { Storage } \\
\text { (months) }\end{array}$ & TVC & $\begin{array}{c}\text { Lactic Acid } \\
\text { Bacteria }\end{array}$ & $\begin{array}{c}\text { Yeast and } \\
\text { Mold }\end{array}$ \\
\hline \multirow{4}{*}{$\begin{array}{c}\text { Basic } \\
\text { Keang-hlueng } \\
\text { Paste AT }\end{array}$} & 0 & $1.89 \times 10^{3}$ & $3.10 \times 10^{2}$ & $<30$ \\
\hline & 2 & $4.70 \times 10^{3}$ & $9.70 \times 10^{2}$ & $<30$ \\
\hline & 4 & $4.30 \times 10^{3}$ & $6.20 \times 10^{2}$ & $<30$ \\
\hline & 6 & $8.00 \times 10^{3}$ & $3.00 \times 10^{2}$ & $<30$ \\
\hline \multirow{4}{*}{$\begin{array}{c}\text { Basic } \\
\text { Keang-hleung } \\
\text { Paste } 4^{\circ} \mathrm{C}\end{array}$} & 0 & $1.89 \times 10^{3}$ & $3.10 \times 10^{2}$ & $<30$ \\
\hline & 2 & $8.70 \times 10^{2}$ & $3.20 \times 10^{2}$ & $<30$ \\
\hline & 4 & $1.28 \times 10^{3}$ & $4.80 \times 10^{2}$ & $<30$ \\
\hline & 6 & $7.60 \times 10^{3}$ & $<30$ & $<30$ \\
\hline \multirow{4}{*}{$\begin{array}{c}\text { Garcinia } \\
\text { Keang-hlueng } \\
\text { Paste AT }\end{array}$} & 0 & $6.60 \times 10^{2}$ & $<30$ & $<30$ \\
\hline & 2 & $4.70 \times 10^{2}$ & $<30$ & $<30$ \\
\hline & 4 & $3.00 \times 10^{3}$ & $<30$ & $<30$ \\
\hline & 6 & $2.13 \times 10^{3}$ & $<30$ & $<30$ \\
\hline \multirow{4}{*}{$\begin{array}{c}\text { Garcinia } \\
\text { Keang-hlueng } \\
\text { Paste } 4^{\circ} \mathrm{C}\end{array}$} & 0 & $6.60 \times 10^{2}$ & $<30$ & $<30$ \\
\hline & 2 & $5.30 \times 10^{2}$ & $<30$ & $<30$ \\
\hline & 4 & $600 \times 10^{2}$ & $<30$ & $<30$ \\
\hline & 6 & $1.71 \times 10^{3}$ & $<30$ & $<30$ \\
\hline \multirow{4}{*}{$\begin{array}{c}\text { Garcinia } \\
\text { Keang-hlueng } \\
\text { Paste } \\
\text { without Salt AT }\end{array}$} & 0 & $1.95 \times 10^{3}$ & $<30$ & $<30$ \\
\hline & 2 & $4.70 \times 10^{2}$ & $3.50 \times 10^{2}$ & $5.20 \times 10^{2}$ \\
\hline & 4 & $7.40 \times 10^{2}$ & $3.00 \times 10^{2}$ & $<30$ \\
\hline & 6 & $1.22 \times 10^{4}$ & $<30$ & $<30$ \\
\hline \multirow{4}{*}{$\begin{array}{c}\text { Garcinia } \\
\text { Keang-hlueng } \\
\text { Paste } \\
\text { without Salt } 4^{\circ} \mathrm{C}\end{array}$} & 0 & $1.95 \times 10^{3}$ & $<30$ & $<30$ \\
\hline & 2 & $5.30 \times 10^{2}$ & $3.20 \times 10^{2}$ & $4.80 \times 10^{2}$ \\
\hline & 4 & $3.50 \times 10^{2}$ & $3.70 \times 10^{2}$ & $<30$ \\
\hline & 6 & $2.53 \times 10^{3}$ & $<30$ & $<30$ \\
\hline
\end{tabular}

$\mathrm{AT}=$ Ambient Temperature.

peratures decreased at 1 mo before kept constant for 3 mo then sharply decreased until got the lowest value compared with other pastes. On the other hand, the FRAP activity of garcinia paste with and without added salt kept in the both storage temperature increased at first 2 mo before kept constant for 3 mo then decreased but still higher than the basic paste (Figures 5 and 6). From the results of DPPH radical scavenging activity and FRAP activity, it pointed out that these parameters of the paste did not have the same change trends at the first 4 mo. This may be a good explanation of using many 


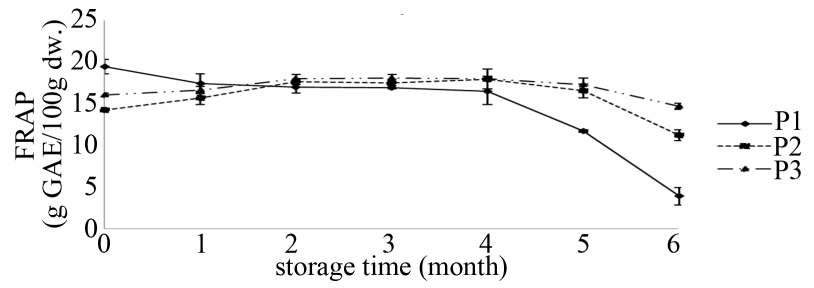

Figure 5. The FRAP activity of Keang-hleung paste during storage at $29^{\circ} \mathrm{C} \pm 2^{\circ} \mathrm{C}$. P1: Basic Keang-hleung paste. P2: Garcinia Keang-hleung paste with $20 \%$ salt. P3: Garcinia Keang-hleung paste without $20 \%$ salt.

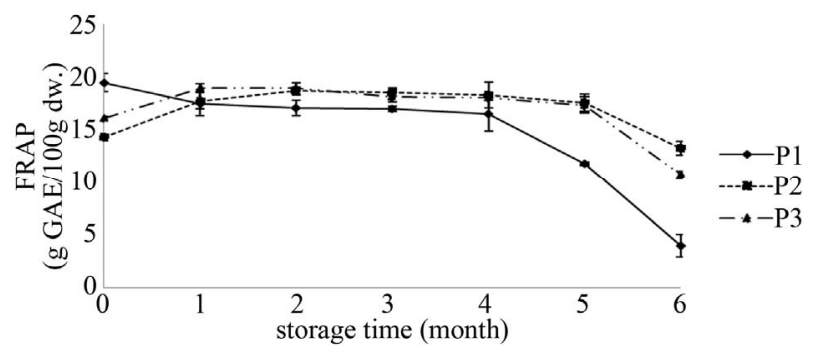

Figure 6. The FRAP activity of Keang-hleung paste during storage at $4^{\circ} \mathrm{C} \pm 2^{\circ} \mathrm{C}$. P1: Basic Keang-hleung paste. P2: Garcinia Keang-hleung paste with $20 \%$ salt. P3: Garcinia Keang-hleung paste without $20 \%$ salt.

assays to evaluate antioxidant activity [29]. However, some researchers mentioned that in vitro antioxidant activity such as DPPH, FRAP, ABTS and metal chelation activity may not responsible for antioxidant activity in vivo or even food system therefore using cellular antioxidant activity assay was more useful and close to body system $[28,30]$.

\subsection{Microbiological Quality in Basic and Garcinia Keang-hleung Paste during Storage at Ambient Temperature $\left(29^{\circ} \mathrm{C} \pm 2^{\circ} \mathrm{C}\right)$ and $4^{\circ} \mathrm{C} \pm 2^{\circ} \mathrm{C}$}

The basic and garcinia Keang-hleung paste with and without salt stored at ambient temperature and $4^{\circ} \mathrm{C}$ monitored for TVC, yeasts and moulds, staphylococcus aureus, Bacilluus cereus, Lactic acid bacteria, Clostridium perfringens, Salmonella, Escherichia coli and coliforms were showed in Table 6. At the initial stage, TVC of all treatments were in the range of $10^{2}-10^{4} \mathrm{cfu} / \mathrm{g}$ and increased as the storage time increased (Table 6). TVC of any paste kept at both temperatures was not more than $1.22 \times 10^{4} \mathrm{cfu} / \mathrm{g}$ within 6 mo. It pointed out that both salt and garcinia played an important role for microbial growth. However, using certain salt concentration as $20 \%$, garcinia and chilled storage as hurdle parameters seemed to pronounce more inhibitory effect. Yeast and mold counts of the basic and garcinia Keang-hlueng paste with addition salt were under $30 \mathrm{cfu} / \mathrm{g}$ during stor- age. Without salt, yeast and mold counts of the garcinia Keang-hlueng paste increased and reached $10^{2} \mathrm{cfu} / \mathrm{g}$ before declined to lower than $30 \mathrm{cfu} / \mathrm{g}$ at the end of storage. An increase of yeast and mold at the first 2 mo may due to proper germination period of the fungal spore, thereafter a decrease of yeast and mold may due to function of hydroxy acid and other weak acids mainly derived from garcinia. Lactic acid bacteria were lowest in the garcinia paste with added salt. However, it was found that the basic paste had lactic acid bacteria throughout the storage except sample kept at $4^{\circ} \mathrm{C}$ for 6 mo. This result indicated that the salt concentration, garcinia content and storage temperature played their antimicrobial role. It also pointed out that using only salt or garcinia may not enough to control some organism and may stimulate the lactic acid bacteria growth for a period of time. However, there were no Staphylococcus aureus, Bacillus cereus, Clostridium perfringens, Salmonella, Escherichia coli and coliforms detected in all treatments throughout the storage period.

\section{Conclusion}

In general, color values of the basic and garcinia Keanghleung paste kept at ambient temperature and $4^{\circ} \mathrm{C} \pm 2{ }^{\circ} \mathrm{C}$ decreased during storage increased. The $\mathrm{pH}$ values and acidity of all pastes tended to decrease and increase, respectively when storage time increased. A decrease of total phenolic content of during storage at ambient and $4^{\circ} \mathrm{C} \pm 2{ }^{\circ} \mathrm{C}$ of the paste was found particularly in the garcinia paste without added salt. A decrease of total phenollic content of the basic paste was concomitant with a decrease of DPPH radical scavenging and FRAP activity. However, there was not a good relationship between DPPH radical scavenging activity and FRAP activity in the garcinia paste with and without added salt. Salt and garcinia in the paste help to prolong to the shelf-life of the paste in term of microbiological quality.

\section{Acknowledgements}

The authors would like to thanks the National Research University Project of Thailand's Office of the Higher Education Commission, Nutraceutical and Functional Food Research and Development Center (NFFRDC) and the Graduated School, University Research Funding AGR540561S, Prince of Songkla University for the financial support.

\section{REFERENCES}

[1] I. O. Aruoma, "Free Radicals, Oxidative Stress and Antioxidants in Human Health and Disease," Journal of the American Oil Chemists Society, Vol. 75, No. 2, 1998, pp. 199-212. doi:10.1007/s11746-998-0032-9 
[2] A. J. Ruby, G. Kuttan, K. D. Baru, K. N. Rajasekharan and R. Kuttan, "Anti-tumour and Antioxidant Activity of Natural Curcuminiods," Cancer Letter, Vol. 94, No.1, 1995, pp. 79-83. doi:10.1016/0304-3835(95)03827-J

[3] H. Ahsan, N. Parveen, U. K. Nizam and S. M. Hadi, "Pro-Oxidant, Anti-Oxidant and Cleavage Activities on DNA of Curcumin and Its Derivatives Demethoxycurcumin and Bisdemethoxycurcumin," Chemico-Biological Interactions, Vol. 121, No. 2, 1999, pp. 161-175. doi:10.1016/S0009-2797(99)00096-4

[4] G. K. Jayaprakasha, L. J. Rao and K. K. Sakariah, “Antioxidant Activities of Curcumin, Demethoxycurcumin and Bisdemethoxycurcumin," Food Chemistry, Vol. 98, No. 4, 2006, pp. 720-724. doi:10.1016/j.foodchem.2005.06.037

[5] M. Cousins, J. Adelberg, F. Chen and J. Rieck, "Antioxidant Capacity of Fresh and Dried Rhizomes from Four Clones of Turmeric (Curcuma longa L.) Grown in Vitro," Industrial Crops and Products, Vol. 25, No. 2, 2007, pp. 125-139. doi:10.1016/j.indcrop.2006.08.004

[6] K. Hayamizu, Y. Ishii, I. Kaneko, M. Shen, Y. Okuhara, N. Shigematsu, H. Tomi, M. Furuse, G. Yoshino and H. Shimasaki, "Effect of Garcinia Cambogia (Hydroxycitric acid) on Visceral Fat Accumulation: Adouble-Blind, Randomized, Placebo-Controlled Trial," Current Therapeutic Research, Vol. 64, No. 8, 2003, pp. 551-567. doi:10.1016/j.curtheres.2003.08.006

[7] M. G. Soni, G. A. Burdock, H. G. Preuss, S. J. Stohs, S. E. Ohia and D. Bagchi, "Safety Assessment of (-)-Hydroxycitric acid and Super Citrimaax, a Novel Calcium/Potassium Salt," Food and Chemical Toxicology, Vol. 42, No. 9, 2004, pp. 1513-1529.

doi:10.1016/j.fct.2004.04.014

[8] S. Siripongvutikorn, C. Thongraung, W. Usawakesmanee, T. Buatoom and P. Thammarutwasik, "Development of Instant Garcinia (Garcinia atroviridis) Tum-Yum Mix as a High Acid Seasoning," Journal of Food Processing and Preservation, Vol. 33, No. 1, 2009, pp. 74-86. doi:10.1111/j.1745-4549.2008.00238.x

[9] A. P. Bartolomé, P. Ruperéz and C. Fúster, "Pineapple Fruit: Morphological Characteristics, Chemical Composition and Sensory Analysis of Red Spanish and Smooth Cayenne Cultivars," Food Chemistry, Vol. 53, No. 1, 1995, pp. 75-79. doi:10.1016/0308-8146(95)95790-D

[10] AOAC, "Official Analytical Chemists," 16th Edition, The Association of Official Analytical Chemists, Inc., Washington DC, 1999.

[11] M. P. Kahkonen, A. I. Hopia, H. J. Vuorela, J. P. Rauha, K. Pihlaja, T. S. Kujala and M. Heinonen, "Anioxidant Activity of Plant Extracts Containing Phenolic Compounds," Journal of Agricultural and Food Chemistry, Vol. 47, No. 10, 1999, pp. 3954-3962. doi:10.1021/jf9901461

[12] H. C. Wu, H. M. Chen and C. Y. Shiau, "Free Amino Acid and Peptide as Related to Antioxidant Properties in Protein Hydrolysates of Mackerel (Scomber austriasicus)," Food Research International, Vol. 36, No. 9-10, 2003, pp. 949-957. doi:10.1016/S0963-9969(03)00104-2

[13] I. F. F. Benzie and J. J. Strain, "The Ferric Reducing
Ability of Plasma (FRAP) as a Measure of 'Antioxidant Power': The FRAP Assay," Analytical Biochemistry, Vol. 239, No. 292, 1996, pp. 70-76. doi:10.1006/abio.1996.0292

[14] U.S. Department of Health and Human Service, U.S. Food and Drug Administration, Center of Food Safety and Applied Nutrition, "Bacteriological Analytical Manual," 2001 .

http://www.fda.gov/Food/FoodScienceResearch/Laborato ryMethods/ucm114664.htm

[15] P. Voroquaux and R. C. Wiley, "Biological and Biochemical Changes in Mimimally Processed Refrigerated Fruits and Vegetables," In: R. C. Wiley, Ed., Minimally Processed Refrigerated Fruits and Vegetables, Chapman and Hall, New York, 1994, pp. 226-268. doi:10.1007/978-1-4615-2393-2 6

[16] O. Lamikanra and M. A. Watson, "Cantaloupe Melon Peroxidase: Characterization and Effects of Additives on Activity," Molecular Nutrition and Food Research, Vol. 44, No. 3, 2000, pp. 168-172.

[17] S. Ketsa and S. Pangkool, "The Effect of Humidity on Ripening of Durians," Postharvest Biology and Technology, Vol. 4, No. 1-2, 1994, pp. 159-165. doi:10.1016/0925-5214(94)90017-5

[18] S. Ketsa and S. Pangkool, "The Effect of Temperature and Humidity on the Ripening of Durian Fruits," Journal of Horticultural Science, Vol. 70, No. 5, 1995, pp. 827831.

[19] G. W. Schieffer, "Pressurized Liquid Extraction of Curcuminoids and Curcuminoid Degradation Products from Turmeric (Curcuma longa) with Subsequent HPLC Assays," Journal of Liquid Chromatography and Related Technologies, Vol. 25, No. 19, 2002, pp. 3033-3044. doi:10.1081/JLC-120015889

[20] A. Concellón, M. C. Anón and A. R. Chaves, "Characterization and Changes in Polyphenol Oxidase from Eggplant Fruit (Solanum melongena L.) during Storage at Low Temperature," Food Chemistry, Vol. 88, No. 1, 2004, pp. 17-24. doi:10.1016/j.foodchem.2004.01.017

[21] T. Anguelova and J. Warthesen, "Degradation of Lycopene, $\alpha$-Carotene, and $\beta$-Carotene during Lipid Peroxidation," Food Chemistry and Toxicology, Vol. 65, No. 1, 2000, pp. 71-75.

[22] L. Axelsson, "Lactic Acid Bacteria : Classification and Physiology," In: S. Salminen, A. V. Wright and A. Ouwehand, Eds., Lactic Acid Bacteria Microbiological and Functional Aspects, Marcel Dekker, New York, 2004, pp. 1-16. doi:10.1201/9780824752033.ch1

[23] M. Maki, "Lactic Acid Bacteria in Vegetable Fermentations," In: S. Salminen, A. V. Wright and A. Ouwehand, Eds., Lactic Acid Bacteria Microbiological and Functional Aspects, Marcel Dekker, New York, 2004, pp. 419-430. doi:10.1201/9780824752033.ch14

[24] M. Sautour, C. S. Mansur, C. Divies, M. Bensoussan and P. Dantigny, "Comparison of the Effects of Temperature and Water Activity on Growth Rate of Food Spoilage Moulds," Journal of Industrial Microbiology and Biotechnology, Vol. 28, No. 6, 2002, pp. 311-315. 
doi:10.1038/si.jim.7000248

[25] T. Hanato, H. Kagawa, T. Yasuhara and T. Okuda, "Two New Flavonoids and Other Constituents in Licorice Root: Their Relative Astringency and Radical Scavenging Effects," Chemical and Pharmaceutical Bulletin, Vol. 36, No. 6, 1988, pp. 2090-2097. doi:10.1248/cpb.36.2090

[26] R. Amarowicz, R. B. Pegg, P. Rahimi-Moghaddam, B. Barl and J. A. Weil, "Free Radical Scavenging Capacity and Antioxidant Activity of Selected Plant Species from the Canadian Prairies," Food Chemistry, Vol. 84, No. 4, 2004, pp. 551-562. doi:10.1016/S0308-8146(03)00278-4

[27] B. Bozin, N. M. Dukic, I. Samojlic, A. Goran and R. Igic, "Phenolics as Antioxidants in Garlic (Allium sativum L., Alliaceae)," Food Chemistry, Vol. 111, No. 4, 2008, pp.
925-929. doi:10.1016/j.foodchem.2008.04.071

[28] K. L. Wolfe and R. H. Liu, "Structure Activity Relationships of Flavonoids in the Cellular Antioxidant Activity Assay," Journal of Agricultural and Food Chemistry, Vol. 56, No. 18, 2008, pp. 8404-8411. doi:10.1021/jf8013074

[29] G. Du, M. Li and F. Ma, "Antioxidant Capacity and the Relationship with Polyphenol and Vitamin C in Actinidia fruits," Food Chemistry, Vol. 113, No. 2, 2009, pp. $557-$ 562. doi:10.1016/j.foodchem.2008.08.025

[30] R. H. Liu and J. Finley, "Potential Potential Cell Culture Models for Antioxidant Research," Journal of Agricultural and Food Chemistry, Vol. 53, No. 10, 2005, pp. 4311-4314. doi:10.1021/jf058070i 